\title{
SIFamide and SIFamide Receptor Define a Novel Neuropeptide Signaling to Promote Sleep in Drosophila
}

\author{
Sangjin Park ${ }^{1,3}$, Jun Young Sonn ${ }^{1,3}$, Yangkyun Oh${ }^{1}$, Chunghun Lim², and Joonho Choe ${ }^{1, *}$
}

\begin{abstract}
SIFamide receptor (SIFR) is a Drosophila G protein-coupled receptor for the neuropeptide SIFamide (SIFa). Although the sequence and spatial expression of SIFa are evolutionarily conserved among insect species, the physiological function of SIFa/SIFR signaling remains elusive. Here, we provide genetic evidence that SIFa and SIFR promote sleep in Drosophila. Either genetic ablation of SIFa-expressing neurons in the pars intercerebralis $(\mathrm{PI})$ or pan-neuronal depletion of SIFa expression shortened baseline sleep and reduced sleep-bout length, suggesting that it caused sleep fragmentation. Consistently, RNA interference-mediated knockdown of SIFR expression caused short sleep phenotypes as observed in SIFa-ablated or depleted flies. Using a panel of neuron-specific Gal4 drivers, we further mapped SIFR effects to subsets of PI neurons. Taken together, these results reveal a novel physiological role of the neuropeptide SIFa/SIFR pathway to regulate sleep through sleep-promoting neural circuits in the PI of adult fly brains.
\end{abstract}

\section{INTRODUCTION}

There are many hypotheses as to why we sleep, but there are three major functions of sleep (Mignot, 2008). First, memory consolidation during sleep occurs by synaptic downscaling (Tononi and Cirelli, 2014). Second, sleep is needed for energy conservation; body temperature in warm-blooded animals goes down during sleep to save energy (Berger and Phillips, 1995), and metabolic activity during sleep is mainly anabolic rather than catabolic (Dworak et al., 2010). Feeding and sleep are two

${ }^{1}$ Department of Biological Sciences, College of Life Science and Bioengineering, Korea Advanced Institute of Science and Technology, Daejeon 305-701, Korea, ${ }^{2}$ Department of Biological Sciences, School of Life Sciences, Ulsan National Institute of Science and Technology, Ulsan $689-798$, Korea, ${ }^{3}$ These authors contributed equally to this work.

*Correspondence: jchoe@ kaist.ac.kr

Received 16 December, 2013; revised 25 February, 2014; accepted 4 March, 2014; published online 21 March, 2014

Keywords: Drosophila melanogaster, Pars Intercerebralis, sleep, SIFamide, SIFamide receptor conflicting but interrelated behaviors important for homeostasis, as sleep is suppressed by starvation both in flies and mammals (Keene et al., 2010; Macfadye et al., 1973), whereas sleep deprivation promotes feeding behaviors (Rechtschaffen and Bergmann, 2002). Lastly, vital cellular components, such as those in immune system, are recovered during sleep (Besedovsky et al., 2012). Despite essential roles of sleep in daily physiology, it remains unclear how sleep processes are maintained and regulated at the levels of genes and neural pathways. Drosophila melanogaster has been a powerful genetic tool to understand the molecular and neuronal bases underlying animal behaviors. Fly sleep shares all of the fundamental traits of mammalian sleep except the sleeping posture and some neurochemistry (e.g., no norephinephrine, no hypocretin/orexin) (Cirelli, 2009; Hendricks et al., 2000; Shaw et al., 2000). Moreover, genetic screens in flies have successfully identified novel sleep genes and neural centers to shape sleep architecture. Multiple brain compartments, such as the mushroom body (MB), fan-shaped body (FB), lateral ventral neurons, and the pars intercerebralis $(\mathrm{PI})$ have been shown to regulate sleep (Foltenyi et al., 2007; Joiner et al., 2006; Liu et al., 2012; Parisky et al., 2009; Pitman et al., 2006). Notably, the PI in flies is endocrinologically equivalent to the hypothalamus-pituitary system in vertebrates and has neuron subsets that promote either arousal or sleep (Crocker and Sehgal, 2010).

In the course of our behavioral screens to identify novel sleep-regulatory genes and neurons, we found evidence that SIFamide (SIFa)-expressing neurons promote sleep. The SIFa family was initially isolated as a myotropic peptide from the grey fleshfly Neobellieria bullata (Janssen et al., 1996). The structure of the mature form of SIFa (AYRKPPFNGSIFamide) and its distribution are well conserved in many insect species (Broeck, 2001; Verleyen et al., 2004). Drosophila SIFa is strongly expressed in four interneurons located in the $\mathrm{PI}$ and regulates sexual behaviors (Terhzaz et al., 2007; Verleyen et al., 2004). Moreover, they project to the fan-shaped body and innervate the surface of the ellipsoid body and the protocerebral bridge (Kahsai and Winther, 2011). SIFa specifically activates the G protein-coupled SIFa receptor, SIFR (Jorgensen et al., 2006). Although the function of SIFR is not well understood, recent reports suggest that it has roles in heat nociception (Neely et al., 2010), longevity (Paik et al., 2012), and calcium signaling (Agrawal et al., 2013). In this study, we show that SIFa and SIFR coordinately regulate sleep through SIFa-expressing and sleep- 
A

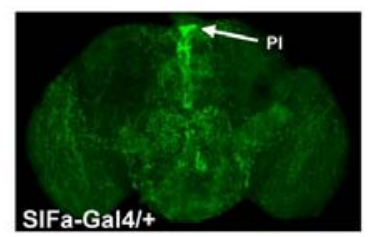

C
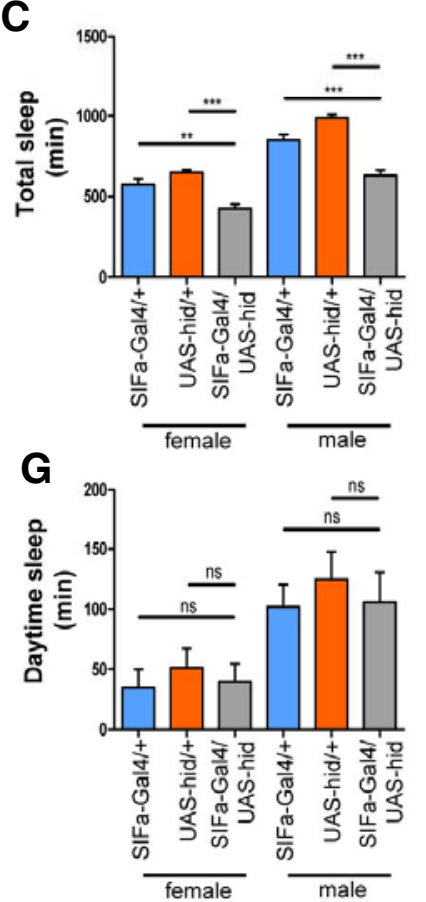
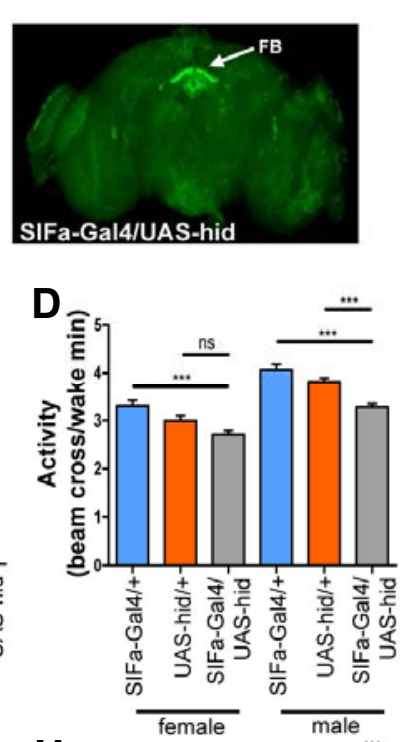

H

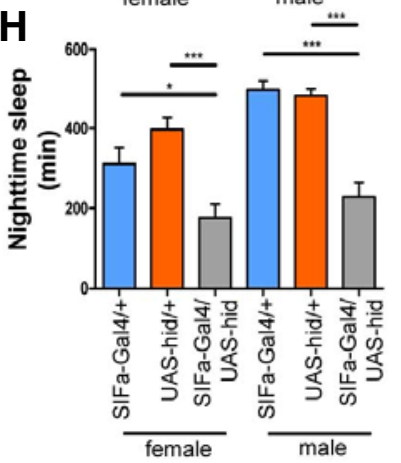

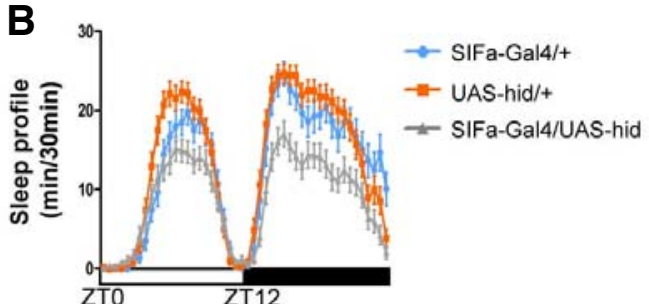

E
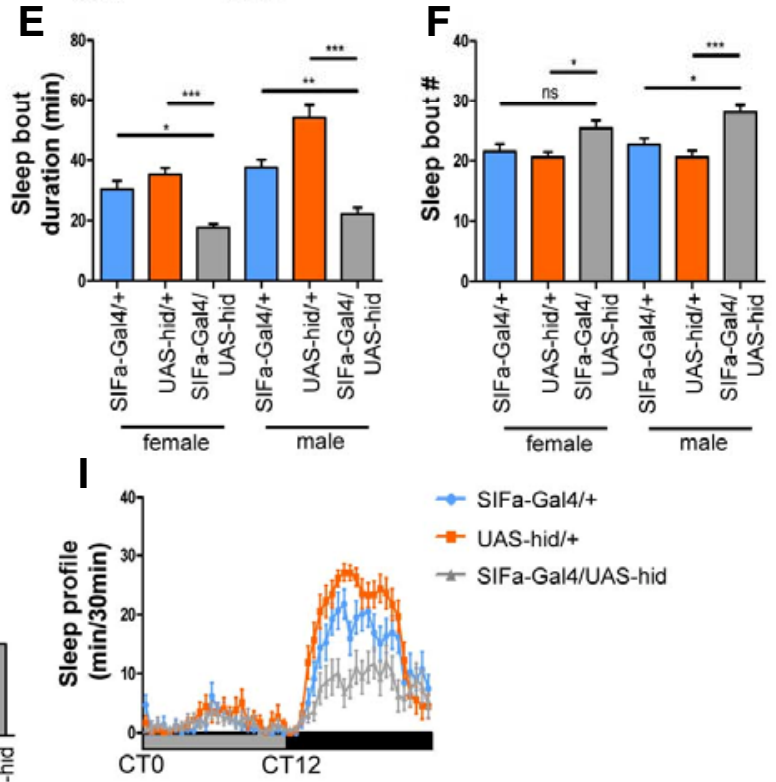

- SIFa-Gal4/+

- UAS-hid/+

- SIFa-Gal4/UAS-hid

Fig. 1. Ablation of SIFa-expressing PI neurons shortens baseline sleep. (A) SIFa peptide diminishes in SIFa-expressing PI neurons when the apoptosis-inducing gene, head involution defective (hid) was ectopically expressed by SIFa-Gal4. Adult fly brains were dissected and labeled with an anti-SIFa antibody and FITC-conjugated secondary antibody (green). PI, pars intercerebralis; FB, fan-shaped body. (B-I) Baseline sleep is shortened and fragmented in both male and female flies with the ablation of SIFa-expressing PI neurons. Locomotor activities from individual flies were recorded in 12:12-h LD or DD cycles. Sleep profile $(B, I)$, total sleep time $(C, G$, and $H)$, activity level during wake $(D)$, sleep bout duration $(E)$, and sleep bout number $(F)$ were calculated and averaged per each genotype. Error bars indicate standard error of mean (SEM). One-way analysis of variance (ANOVA) followed by Bonferroni's Multiple Comparison test was used for statistical analysis. ${ }^{*} p<$ $0.05 ;{ }^{* *} p<0.01 ;{ }^{* \star *} p<0.001 ;$ ns, not significant.

promoting neurons in the $\mathrm{PI}$, respectively, thus revealing a novel neuropeptide signaling pathway in Drosophila sleep.

\section{MATERIALS AND METHODS}

Fly strains and culture

All flies were maintained on a 12-h light-dark cycle on standard cornmeal-yeast-agar medium at $25^{\circ} \mathrm{C}$ and $60 \%$ humidity. ElavGal4;UAS-Dicer2 (25750), c309-Gal4 (6906), 201y-Gal4 (4440), 1471-Gal4 (9465), c205-Gal4 (30826), C5-Gal4 (30839), GH146Gal4 (30026), GH298-Gal4 (37294), SG18.1-Gal4 (6405), GMRGal4 (9146), Dilp2-Gal4 (37516), 121y-Gal4 (30815), and c767Gal4 (30848) were obtained from the Bloomington Drosophila Stock Center (USA). UAS-SIFR RNAi (10823R-1) was purchased from NIG-Fly (Japan). SIFa-Gal4 and UAS-SIFa RNAi fly lines were a kind gift from Dr. J. A. Veenstra (University of Bordeaux). To construct UAS-SIFR, the SIFR coding sequence was cloned into a UAS vector, pSST13, derived from the
pKC27 donor plasmid, which uses the ФC31 site-specific integration system (Groth et al., 2004). The transgene was inserted into the VIE-72a site located on the second chromosome.

\section{Quantitative PCR}

Total RNA was isolated using TRIzol (Invitrogen) from 50 adult fly heads per genotype. After DNase I treatment (Promega), 2 $\mu \mathrm{g}$ of total RNA was reverse-transcribed using TOPscript ${ }^{\mathrm{TM}} \mathrm{RT}$ DryMIX (Enzynomics) and oligo-(dT) 18 primer. Relative transcript levels of SIFR and Rp49 genes were quantified by realtime PCR using TOPreal ${ }^{\mathrm{TM}}$ qPCR 2× PreMIX (SYBR Green, Enzynomics) and iQ5 system (Bio-Rad). The specific primer sequences used in our study were 5'- AAT GAA ACC ATA CGC AAA CCC -3' and 5'- CTG GAT AAC TGC CTC AGA CTG -3' for SIFR and 5'- GAA GAA GCG CAC CAA GCA CT $3^{\prime}$ and 5'- TTG AAT CCG GTG GGC AGC AT -3' for Rp49 as a control. 
A
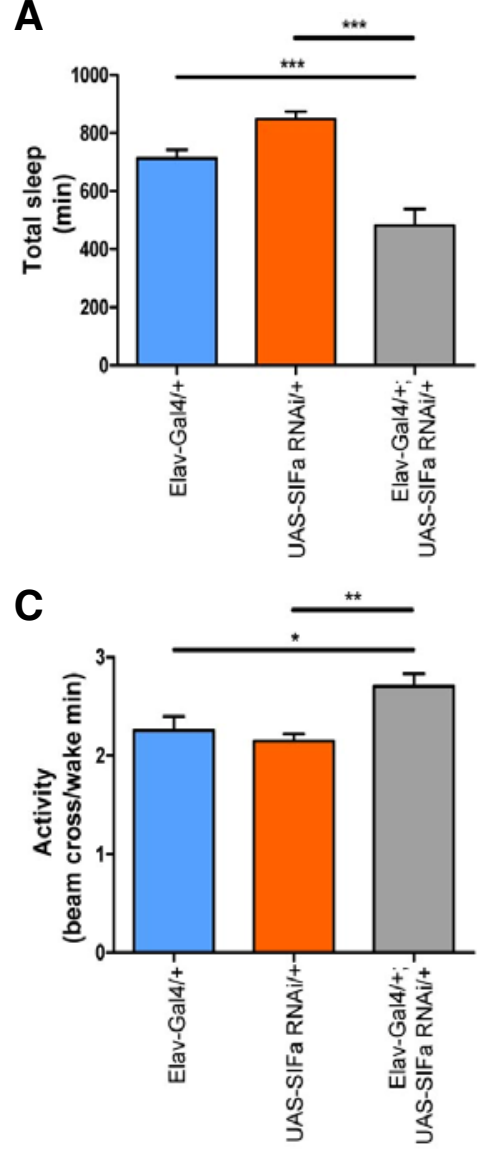

B

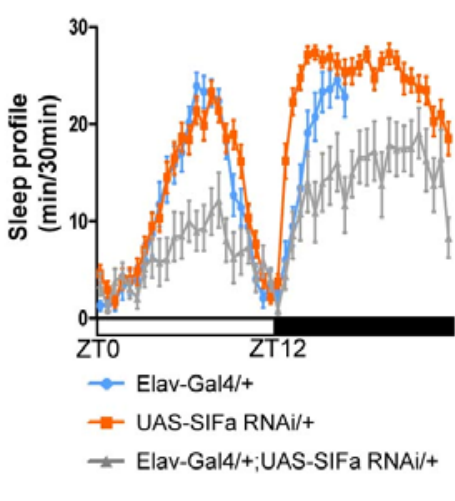

D

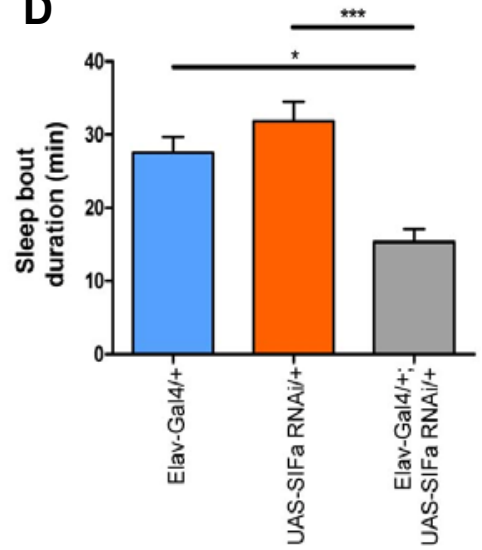

Fig. 2. Pan-neuronal depletion of SIFa neuropeptide mimics the ablation of SIFa-expressing neurons to decrease sleep. The SIFa RNAi transgene was expressed with a pan-neuronal Elav-Gal4 driver. Locomotor activities were recorded from individual female flies in LD cycles as in Fig. 1. Total sleep time (A), sleep profile (B), activity level during wake (C), and sleep bout duration (D) were calculated and averaged per each genotype. Error bars indicate SEM. One-way ANOVA followed by Bonferroni's Multiple Comparison test was used for statistical analysis. ${ }^{*} p<0.05,{ }^{* *} p<0.01,{ }^{* \star *} p<0.001$.

\section{Behavioral analyses}

We placed 2-5 day old female flies in $65 \mathrm{~mm} \times 5 \mathrm{~mm}$ glass vials containing $5 \%$ sucrose and $1 \%$ agar. Sleep behavior was monitored using Drosophila Activity Monitors (DAM) (Trikinetics, USA) in a 12-h light/12-h dark chamber kept under constant temperature $\left(25^{\circ} \mathrm{C}\right)$ and humidity $(60 \%)$. Locomotor activity was monitored in 1-min bins. An in-house-developed sleep analysis program was used to analyze sleep parameters (total sleep, wake activity, sleep bout number, and sleep bout duration).

\section{Brain immunohistochemistry}

For anti-SIFa antibody labeling, adult fly brains were dissected in phosphate-buffered saline (PBS) and then fixed in PBS containing $4 \%$ formaldehyde. Brains were blocked with $3 \%$ normal goat serum in PBS containing $0.3 \%$ Triton X-100 (PBS-T) and then incubated overnight at $4^{\circ} \mathrm{C}$ with anti-SIFa serum (a kind gift from Dr. J. A. Veenstra) diluted in the blocking solution at 1:1,000. After washing with PBS-T, brains were incubated with fluorescein isothiocyanate (FITC)- or rhodamine-conjugated anti-rabbit secondary antibodies (Jackson ImmunoResearch) diluted in PBS-T at 1:1,000. Brains were mounted in VECTASHIELD Mounting Medium (Vector Laboratories, USA) and examined by confocal microscopy (LSM710).

\section{RESULTS}

Ablation of SIFa-expressing neurons shortens baseline sleep To test if SIFa-expressing neurons regulate sleep behaviors, we genetically ablated SIFa-expressing neurons in transgenic flies using the Gal4/UAS system (Southall et al., 2008) and recorded their daily baseline sleep in light-dark (LD) conditions. SIFa is a neuropeptide expressed in four neurons within the PI and has been shown to modulate sexual behaviors in flies (Terhzaz et al., 2007). We confirmed that SIFa peptide was not detectable in SIFa-expressing PI neurons when the apoptosisinducing gene, head involution defective (hid), was ectopically expressed by SIFa-Gal4 (Fig. 1A). Notably, ablation of SIFaexpressing PI neurons increased anti-SIFa antibody labeling in the $\mathrm{FB}$, indicating a possible in vivo compensation mechanism. Nonetheless, ablation of these four neurons significantly decreased total sleep amounts in both male and female flies as compared to SIFa-Gal4/+ and UAS-hid/+ control flies (Figs. 1B and $1 \mathrm{C}, p<0.01$ ).

To determine whether general hyperactivity led to the short sleep phenotype in SIFa-ablated flies, we examined locomotor activity during their wake state. As shown in Fig. 1D, wake activity was decreased by the ablation of SIFa-expressing PI neurons compared to the controls (Fig. 1D, $p<0.001$ ). Hence, shortened sleep in SIFa-ablated flies was not due to their increased activity during the wake state, rather, it is likely that they actually spent less time sleeping. Consistent with this, we observed sleep fragmentation in SIFa-ablated flies; their sleepbout length was reduced to nearly $50 \%$ of controls (Fig. 1E, $p<$ 0.05 ), but the number of sleep bouts was increased (Fig. 1F, $p$ $<0.05$ ). Because environmental factors such as light can influence sleep, we also recorded daily sleep in dark-dark (DD) 

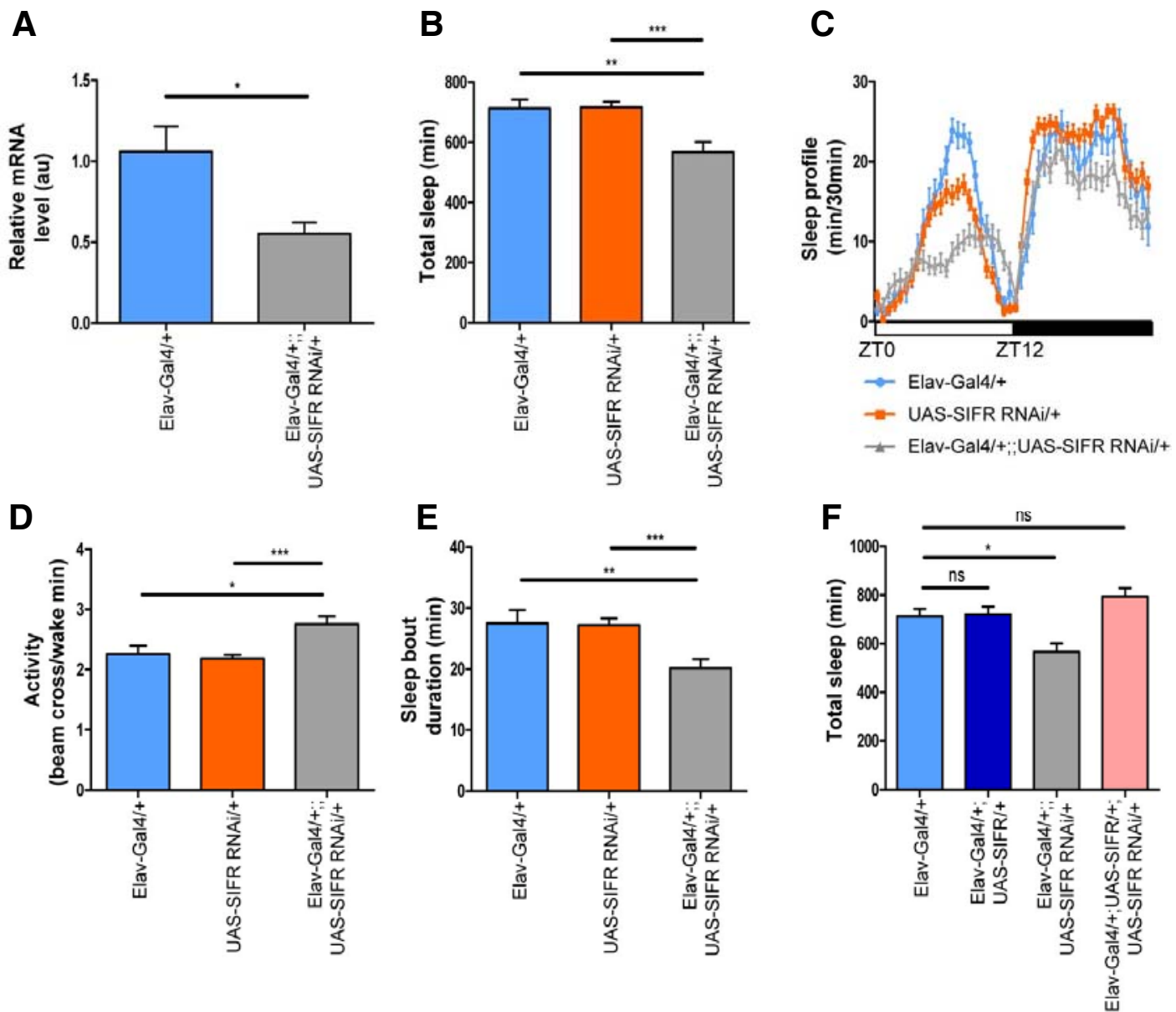

Fig. 3. Pan-neuronal depletion of SIFR causes short sleep. (A) Total RNA was purified from adult fly heads. SIFR mRNA levels were quantified using real-time PCR in triplicate and normalized to Rp49 mRNA levels. Data show the average from three independent experiments. (B-F) SIFR RNAi transgene was expressed with a pan-neuronal Elav-Gal4 driver. Locomotor activities were recorded from individual female flies in LD cycles as in Fig. 1. Total sleep time (B, F), sleep profile (C), activity level during wake (D), and sleep bout duration (E) were calculated and averaged per each genotype. Error bars indicate SEM. Unpaired Student's $t$-tests was used for statistical analysis when test was compared to one control. One-way ANOVA followed by Bonferroni's Multiple Comparison test was used for statistical analysis when tests were compared to two controls. ${ }^{*} p<0.05 ;{ }^{* \star} p<0.01 ;{ }^{* * *} p<0.001$; ns, not significant.

conditions. We found that the short sleep phenotype in SIFaablated flies was particularly evident during the subjective night in DD cycles (Figs. 1G-11, $p<0.05$ ), suggesting that the effects of SIFa-expressing neurons on daily baseline sleep are independent of light.

\section{SIFa depletion mimics the ablation of SIFa-expressing neurons to affect sleep behaviors}

As our genetic ablation strategy revealed a sleep-promoting role of SIFa-expressing neurons, we next investigated whether signaling through the neuropeptide SIFa is directly involved in sleep regulation. To validate this hypothesis, we depleted endogenous expression of SIFa or the SIFa receptor, SIFR, by RNA interference (RNAi) in transgenic flies and examined sleep behaviors.

An SIFa RNAi transgene has been shown to efficiently knock down SIFa expression in PI neurons (Terhzaz et al., 2007). Thus, we expressed the same SIFa RNAi by Elav-Gal4 to completely block SIFa expression in adult fly brains. As shown in Figs. $2 \mathrm{~A}$ and $2 \mathrm{~B}$, SIFa depletion reduced the total sleep amount (Figs. 2A and 2B, $p<0.001$ ) with shortened sleep-bout length (Fig. 2D, $p<0.05$ ), largely mimicking the sleep phenotypes observed in SIFa-ablated flies. This suggests that SIFadepleted flies have difficulties maintaining sleep. However, wake activity in SIFa-depleted flies was higher than that in control flies (Fig. 2C, $p<0.05$ ). Nonetheless, SIFa RNAi flies showed similar locomotor activity to the SIFa-ablated flies. Therefore, it is less likely that SIFa depletion-induced hyperactivity is sufficient to explain the perturbations in sleep behavior.

\section{Pan-neuronal depletion of SIFR causes short sleep}

When SIFR expression was knocked down using the panneuronal Elav-Gal4 driver, we consistently observed a significant reduction in total sleep amount (Fig. 3B, $p<0.01$ ). The short sleep in SIFR-depleted flies was more evident during the light phase (i.e., daytime) of LD cycles (Fig. $3 C$ ), possibly due to a modest decrease in SIFR mRNA levels by the pan-neuronal expression of the SIFR RNAi transgene (Fig. 3A, $p<0.05$ ) and/or a non-neuronal role of SIFR on sleep regulation. Similarly to SIFa-depleted flies, wake activity was significantly in- 

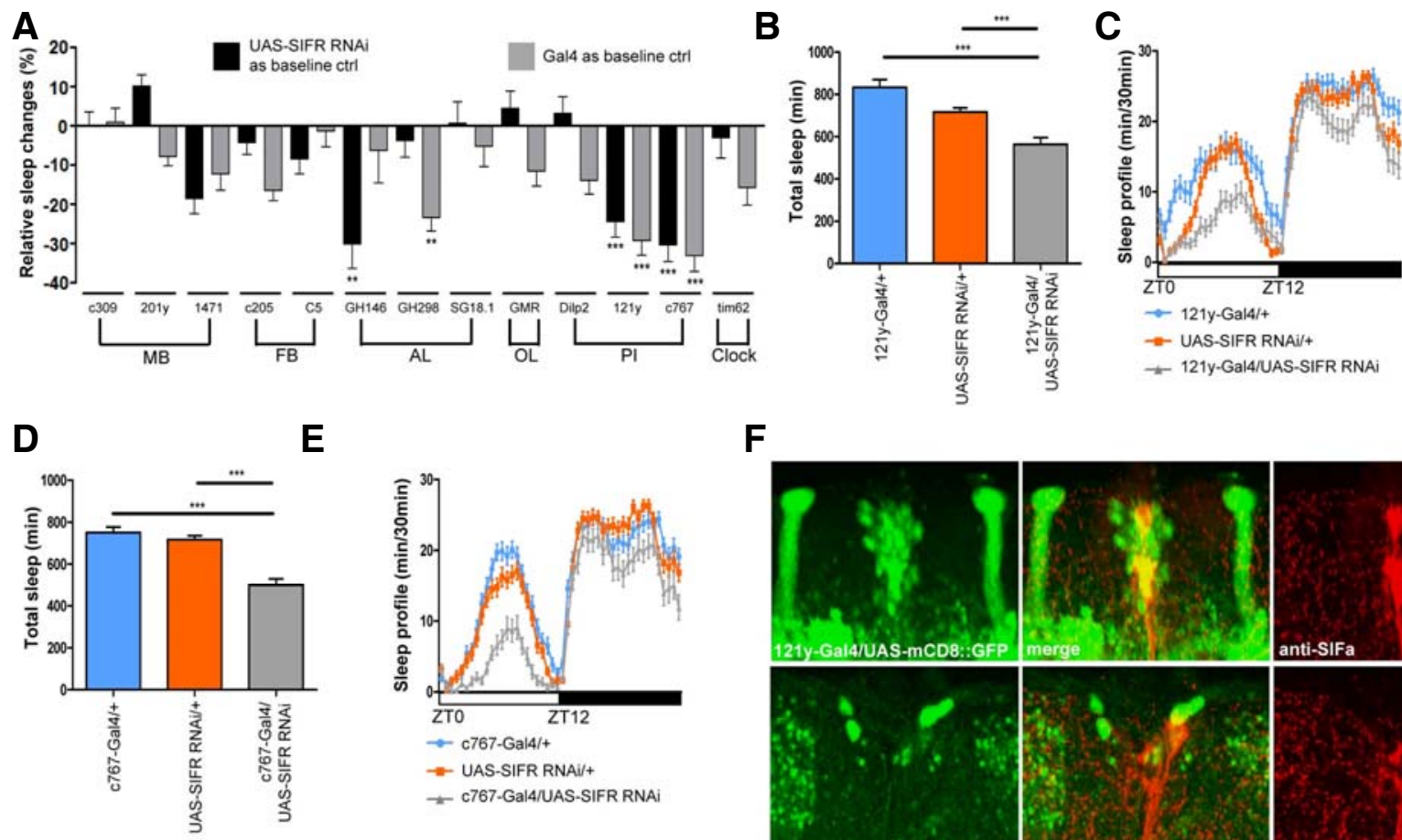

$\mathbf{E}$

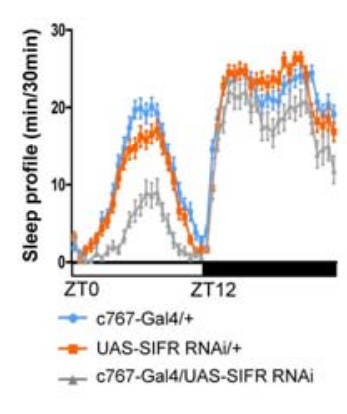

$\mathbf{F}$

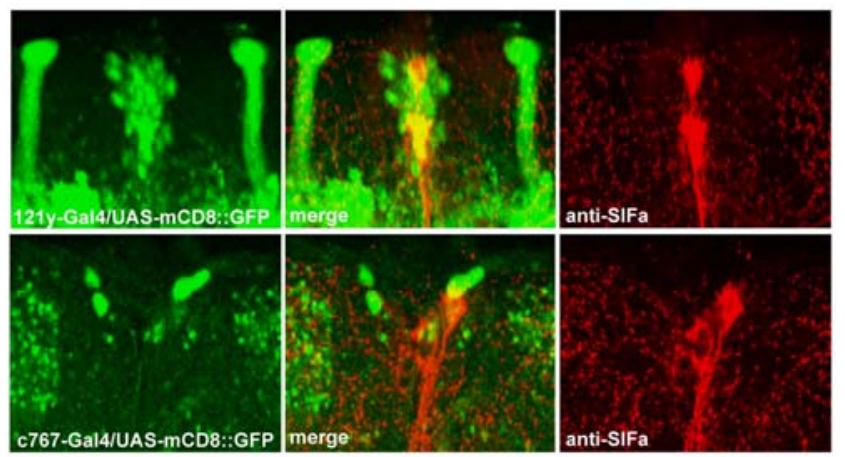

Fig. 4. SIFR knockdown in particular subset neurons of the PI decreases sleep. (A) SIFR was depleted in various regions of fly brain, including the mushroom body $(\mathrm{MB})$, fan-shaped body $(\mathrm{FB})$, antennal lobe $(\mathrm{AL})$, optic lobe $(\mathrm{OL})$, pars intercerebralis (PI), and clock neurons. Neuronspecific effects of SIFR depletion on baseline sleep were monitored as in Fig. 1. Sleep change percentages were calculated as compared to the averaged total sleep time in UAS-SIFR RNAi/+ (black bars) or Gal4/+ (gray bars) control flies. (B, C) Total sleep time and sleep profile when SIFR expression was depleted using the 121y-Gal4 driver. (D, E) Total sleep time and sleep profile when SIFR expression was depleted using the $c 767-G a l 4$ driver. (F) Expression patterns of $121 y$-Gal4 (top) and c767-Gal4 (bottom) drivers were visualized by GFP expression (green) in adult fly brains. Enlarged PI regions containing SIFa-expressing PI neurons (red) are shown. Error bars indicate SEM. One-way ANOVA followed by Bonferroni's Multiple Comparison test was used for statistical analysis. ${ }^{\star *} p<0.01 ;{ }^{\star \star *} p<0.001$.

creased in SIFR-depleted flies (Fig. 3D, $p<0.05$ ) while their sleep-bout length was reduced (Fig. $3 \mathrm{E}, p<0.01$ ). To confirm the specific effects of SIFR RNAi, we performed a rescue experiment with SIFR overexpression. Pan-neuronal SIFR overexpression itself had no effect on the total sleep time (Fig. 3F), indicating that endogenous levels of SIFR expression are not limiting for baseline sleep. However, when transgenic SIFR was co-expressed with SIFR RNAi, it rescued sleep phenotypes caused by SIFR depletion (Fig. 3F, $p<0.05$ ), excluding the possibility that off-target SIFR RNAi effects led to short sleep observed in SIFR-depleted flies. Taken together, this genetic evidence supports the hypothesis that SIFa/SIFR is a novel neuropeptide signal that promotes sleep in Drosophila.

\section{A subset of PI neurons is important for SIFR-mediated sleep promotion}

To further localize brain regions important for SIFR function in sleep regulation, we screened various Gal4 drivers to express transgenic SIFR RNAi in different groups of neurons, including the $\mathrm{MB}, \mathrm{FB}$, antennal lobe (AL), olfactory lobe $(\mathrm{OL}), \mathrm{PI}$, and circadian-clock neurons (Fig. 4A). SIFR depletion by most of our Gal4 drivers had no significant effects on total sleep amount when compared to both Gal4/+ and UAS-SIFR RNAi/+ control flies. However, daily sleep amount was reduced by $\sim 3 \mathrm{~h}$ when we expressed SIFR RNAi using $121 Y$-Gal4 (Figs. 4B and 4C) or c767-Gal4 (Figs. 4D and 4E). 121Y-Gal4 and c767-Gal4 drivers show distinct expression patterns in adult fly brains, but both of them are expressed in a specific subset of PI neurons adjacent to SIFa-expressing neurons (Fig. 4F). We also tested another PI-specific Gal4 driver, Drosophila insulin-like peptide 2 (Dilp2)-Gal4, and found no significant effect of SIFR depletion in Dilp2-positive PI neurons on baseline sleep. As insulinsecreting PI neurons have been shown to mediate octopaminedependent wake-promoting signals (Crocker et al., 2010) (also see "Discussion"), our data support a novel sleep-promoting role of the SIFa/SIFR pathway in Dilp2-negative, SIFR-positive PI neurons.

\section{DISCUSSION}

Our study provides new evidence that the neuropeptide SIFa and its G protein-coupled receptor SIFR are novel mediators for promoting sleep in Drosophila. We found that either genetic ablation of SIFa-expressing neurons or depletion of SIFa expression shortened baseline sleep and caused sleep fragmentation by decreasing sleep-bout length. Consistent with our observation, a recent study independently revealed a possible sleep promoting role of SIFa-expressing neurons in DD conditions (Shang et al., 2013). Using neuron-specific SIFR depletion, we further mapped the sleep-promoting SIFR function to 
Dilp2-negative, SIFR-positive PI neurons.

The PI in adult fly brain is homologous to the mammalian hypothalamus, the control center for neurotransmitter regulation. Several therapeutic targets for human sleep disorders are concentrated in the hypothalamus (Mignot et al., 2002). For instance, dopaminergic neurons blocked by amphetamine-like drugs induce wake-promoting signals to cure narcolepsy (Wisor et al., 2001). Benzodiazepine compounds increase gammaaminobutryic acid (GABA)ergic neuronal transmission to enhance sleep-promoting signals to treat insomnia (Smith, 2001). Octopamine, which is similar to mammalian norepinephrine (Roeder, 1999), has been identified as a wake-promoting molecule in Drosophila (Crocker and Sehgal, 2008; Crocker et al., 2010). When octopamine biosynthesis is compromised, flies exhibit enhanced sleep. On the other hand, octopamine promotes wakefulness in flies, particularly at night (Crocker and Sehgal, 2008). Moreover, octopamine and OAMB, an octopamine receptor, act in Dilp2-expressing PI neurons to promote wakefulness through the cyclic AMP (cAMP) pathway (Crocker et al., 2010). This is in contrast with our finding that Dilp2negative PI neurons are important for SIFR-dependent sleep promotion. Therefore, we defined a novel PI circuit that promotes sleep via the SIFa-SIFR signaling pathway.

Additional genes have been identified as sleep regulators in the PI region of adult fly brain, including members of the rhomboid family, which are integral membrane proteases and star, a transmembrane cargo receptor. They process epidermal growth factor receptor (EGFR)- activating ligands, such as spitz, gurken, and keren, so that extracellular signal-regulated kinase (ERK) is activated by phosphorylation. When EGFR is activated, flies exhibit excessive sleep (Foltenyi et al., 2007). Interestingly, depletion of rhomboid, one of the processors for the ligand of the EGF receptor in c767-Gal4 expressing PI neurons shortened sleep (Foltenyi et al., 2007). Given that SIFR and rhomboid promote sleep in the same $\mathrm{PI}$ neurons, it might be possible that SIFR and rhomboid function together to regulate sleep through the EGFR-ERK signaling pathway. Not much is known about the SIFR in terms of its downstream effectors and how it exerts its physiological effects. In general, GPCR activates the protein kinase A (PKA)-cAMP pathway via Gs or $\mathrm{Ca}^{2+}$ through a $\mathrm{Gq}$ regulator (Selbie and Hill, 1998). It was recently shown that lethality in flies with SIFR knock-down is rescued by the overexpression of dSTIM, one of the key regulators of storeoperated $\mathrm{Ca}^{2+}$ entry (Agrawal et al., 2013). Furthermore, the nuclear factor of activated T cells (NFAT), a $\mathrm{Ca}^{2+}$-activated transcription factor, is regulated by SIFR in a Schneider 2 (S2) cell-based dsRNA screening (Gwack et al., 2006), suggesting that sleep regulation by SIFR might involve $\mathrm{Ca}^{2+}$ signaling. Future studies will address which signaling pathways SIFR affects to regulate neuronal activity and sleep behavior.

\section{ACKNOWLEDGMENTS}

We thank Dr. J. A. Veenstra for the anti-SIFa serum and fly lines (SIFa-Gal4, UAS-SIFa RNAi) and Dr. Y. J. Kim for generating the UAS-SIFR transformants. This work was supported by the 2013 Research Fund (No. 1.130009) of Ulsan National Institute of Science and Technology (UNIST), the 2014 TJ Park Science Fellowship (C.L.), and the National Research Foundation of Korea (No. 20110015442) (J.C.).

\section{REFERENCES}

Agrawal, T., Sadaf, S., and Hasan, G. (2013). A genetic RNAi screen for IP3/Ca ${ }^{2+}$ coupled GPCRs in Drosophila identifies the PdfR as a regulator of insect flight. PLoS Genet. 9, e1003849.
Berger, R.J., and Phillips, N.H. (1995). Energy-conservation and sleep. Behav. Brain Res. 69, 65-73.

Besedovsky, L., Lange, T., and Born, J. (2012). Sleep and immune function. Pflug. Arch. Eur. J. Phy. 463, 121-137.

Broeck, J.V. (2001). Neuropeptides and their precursors in the fruitfly, Drosophila melanogaster. Peptides 22, 241-254.

Cirelli, C. (2009). The genetic and molecular regulation of sleep: from fruit flies to humans. Nat. Rev. Neurosci. 10, 549-560.

Crocker, A., and Sehgal, A. (2008). Octopamine regulates sleep in Drosophila through protein kinase A-dependent mechanisms. J. Neurosci. 28, 9377-9385.

Crocker, A., and Sehgal, A. (2010). Genetic analysis of sleep. Genes Dev. 24, 1220-1235.

Crocker, A., Shahidullah, M., Levitan, I.B., and Sehgal, A. (2010). Identification of a neural circuit that underlies the effects of octopamine on sleep: wake behavior. Neuron 65, 670-681.

Dworak, M., McCarley, R.W., Kim, T., Kalinchuk, A.V., and Basheer, R. (2010). Sleep and brain energy levels: ATP changes during sleep. J. Neurosci. 30, 9007-9016.

Foltenyi, K., Greenspan, R.J., and Newport, J.W. (2007). Activation of EGFR and ERK by rhomboid signaling regulates the consolidation and maintenance of sleep in Drosophila. Nat. Neurosci. 10, 1160-1167.

Groth, A.C., Fish, M., Nusse, R., and Calos, M.P. (2004). Construction of transgenic Drosophila by using the site-specific integrase from phage phi C31. Genetics 166, 1775-1782.

Gwack, Y., Sharma, S., Nardone, J., Tanasa, B., luga, A., Srikanth, S., Okamura, H., Bolton, D., Feske, S., Hogan, P.G., et al. (2006). A genome-wide Drosophila RNAi screen identifies DYRK-family kinases as regulators of NFAT. Nature 441, 646-650.

Hendricks, J.C., Finn, S.M., Panckeri, K.A., Chavkin, J., Williams, J.A., Sehgal, A., and Pack, A.I. (2000). Rest in Drosophila is a sleep-like state. Neuron 25, 129-138.

Janssen, I., Schoofs, L., Spittaels, K., Neven, H., VandenBroeck, J., Devreese, B., VanBeeumen, J., Shabanowitz, J., Hunt, D.F., and DeLoof, A. (1996). Isolation of NEB-LFamide, a novel myotropic neuropeptide from the grey fleshfly. Mol. Cell. Endocrinol. 117, 157-165.

Joiner, W.J., Crocker, A., White, B.H., and Sehgal, A. (2006). Sleep in Drosophila is regulated by adult mushroom bodies. Nature 441, 757-760.

Jorgensen, L.M., Hauser, F., Cazzamali, G., Williamson, M., and Grimmelikhuiizen, C.J. (2006). Molecular identification of the first SIFamide receptor. Biochem. Biophys. Res. Commun. 340, 696701.

Kahsai, L., and Winther, A.M. (2011). Chemical neuroanatomy of the Drosophila central complex: distribution of multiple neuropeptides in relation to neurotransmitters. J. Comp. Neurol. 519, 290-315.

Keene, A.C., Duboue, E.R., McDonald, D.M., Dus, M., Suh, G.S.B., Waddell, S., and Blau, J. (2010). Clock and cycle limit starvationinduced sleep loss in Drosophila. Curr. Biol. 20, 1209-1215.

Liu, Q.L., Liu, S., Kodama, L., Driscoll, M.R., and Wu, M.N. (2012). Two dopaminergic neurons signal to the dorsal fan-shaped body to promote wakefulness in Drosophila. Curr. Biol. 22, 2114-2123.

Macfadye, U.M., Oswald, I., and Lewis, S.A. (1973). Starvation and human slow-wave sleep. J. Appl. Physiol. 35, 391-394.

Mignot, E. (2008). Why we sleep: the temporal organization of recovery. PLoS Biol. 6, e106.

Mignot, E., Taheri, S., and Nishino, S. (2002). Sleeping with the hypothalamus: emerging therapeutic targets for sleep disorders. Nat. Neurosci. 5, 1071-1075.

Neely, G.G., Hess, A., Costigan, M., Keene, A.C., Goulas, S., Langeslag, M., Griffin, R.S., Belfer, I., Dai, F., Smith, S.B., et al. (2010). A genome-wide Drosophila screen for heat nociception identifies alpha 2 delta 3 as an evolutionarily conserved pain gene. Cell $143,628-638$

Paik, D., Jang, Y.G., Lee, Y.E., Lee, Y.N., Yamamoto, R., Gee, H.Y., Yoo, S., Bae, E., Min, K.J., Tatar, M., et al. (2012). Misexpression screen delineates novel genes controlling Drosophila lifespan. Mech. Ageing Dev. 133, 234-245.

Parisky, K.M., Agosto, J., Pulver, S.R., Shang, Y.H., Kuklin, E., Hodge, J.J.L., Kang, K., Liu, X., Garrity, P.A., Rosbash, M., et al. (2009). PDF cells are a GABA-responsive wake-promoting component of the Drosophila sleep circuit (vol. 60, pg. 672-682, 2008). Neuron 61, 152. 
Pitman, J.L., McGill, J.J., Keegan, K.P., and Allada, R. (2006). A dynamic role for the mushroom bodies in promoting sleep in Drosophila. Nature 441, 753-756.

Rechtschaffen, A., and Bergmann, B.M. (2002). Sleep deprivation in the rat: An update of the 1989 paper. Sleep 25, 18-24.

Roeder, T. (1999). Octopamine in invertebrates. Prog. Neurobiol. $59,533-561$

Selbie, L.A., and Hill, S.J. (1998). G protein-coupled-receptor crosstalk: the fine-tuning of multiple receptor-signalling pathways. Trends Pharmacol. Sci. 19, 87-93.

Shang, Y.H., Donelson, N.C., Vecsey, C.G., Guo, F., Rosbash, M., and Griffith, L.C. (2013). Short neuropeptide F is a sleep-promoting inhibitory modulator. Neuron $80,171-183$.

Shaw, P.J., Cirelli, C., Greenspan, R.J., and Tononi, G. (2000) Correlates of sleep and waking in Drosophila melanogaster. Science 287, 1834-1837.

Smith, T.A.D. (2001). Type A gamma-aminobutyric acid (GABA(A)) receptor subunits and benzodiazepine binding: significance to clinical syndromes and their treatment. Br. J. Biomed. Sci. 58, 111-121.

Southall, T.D., Elliott, D.A., and Brand, A.H. (2008). The GAL4 system: a versatile toolkit for gene expression in Drosophila. CSH Protoc. 2008, pdb.top49.

Terhzaz, S., Rosay, P., Goodwin, S.F., and Veenstra, J.A. (2007). The neuropeptide SIFamide modulates sexual behavior in Drosophila. Biochem. Biophys. Res. Commun. 352, 305-310.

Tononi, G., and Cirelli, C. (2014). Sleep and the price of plasticity: from synaptic and cellular homeostasis to memory consolidation and integration. Neuron 81, 12-34.

Verleyen, P., Huybrechts, J., Baggerman, G., Van Lommel, A., De Loof, A., and Schoofs, L. (2004). SIFamide is a highly conserved neuropeptide: a comparative study in different insect species. Biochem. Biophys. Res. Commun. 320, 334-341.

Wisor, J.P., Nishino, S., Sora, I., Uhl, G.H., Mignot, E., and Edgar D.M. (2001). Dopaminergic role in stimulant-induced wakefulness. J. Neurosci. 21, 1787-1794. 